\title{
Experimental Overview of Ultra-Relativistic Heavy lons Physics
}

\section{Paula Bordalo}

Lip

Av. Elias Garcia, 14, Portugal

E-mail: bordalo@lip.pt

\begin{abstract}
A review of some experimental results obtained in ultra-relativistic heavy ion collisions at CERN and at BNL is presented. The choice covers the discussion on main results concerning the electromagnetic and hard probes of quark gluon plasma formation, namely direct and virtual photon production, heavy quarks and quarkonia production, and jets modification in medium.
\end{abstract}




\section{Introduction}

Statistical QCD predicts that, when matter is under conditions of high temperature or pressure, a phase transition will occur forming a new state of matter made of deconfined quarks and gluons - the quark gluon plasma QGP. It is believed that QGP existed at few microseconds after the Big Bang. Lattice QCD calculations give the critical values: a critical temperature $T_{C}$ $\sim 175 \mathrm{MeV}$ and energy densities $\varepsilon_{\mathrm{C}} \sim 1 \mathrm{GeV} / \mathrm{fm}^{3}$ are predicted [1].

The search for QGP formation has been carried on two laboratories CERN and BNL by studying ultra-relativistic heavy ion collisions. Several probes are investigated but, due to a lack of time and space, only a selection on the electromagnetic and on the most penetrating probes will be discussed.

\section{Electromagnetic Probes}

The study of photons production as a probe of quark gluon plasma formation (QGP) is motivated by the fact that they do not interact strongly and so they carry out information about the early stage of the collision.

Thermal photons produced in hot QGP phase will probe its initial temperature. Contrary to early predictions, nowadays it is expected that a hadron gas phase will radiate more photons than QGP.

\subsection{Real Photons}

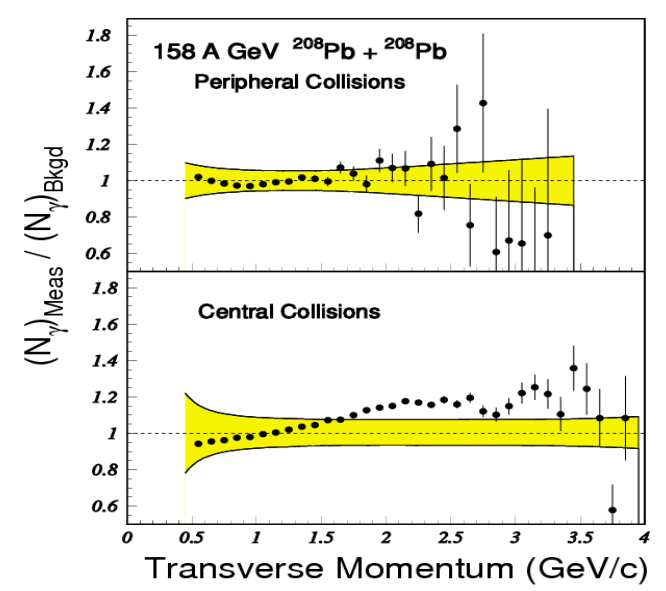

Figure 1. Ratio of photon $\mathrm{p}_{\mathrm{T}}$ distributions measured over background obtained by WA98 experiment.

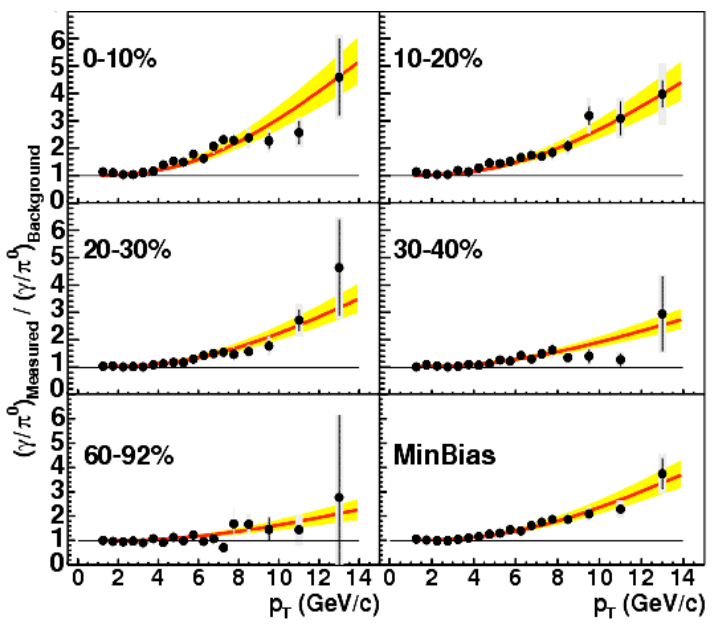

Figure 2. PHENIX results on the $\gamma / \pi^{0}$ ratios, measured over the calculated background, as a function of $\mathrm{p}_{\mathrm{T}}$ for different $\mathrm{Au}-\mathrm{Au}$ centralities.

The first SPS/CERN generation experiments [2] at $\sqrt{ } \mathrm{s}_{\mathrm{nn}}=19.4 \mathrm{GeV}$ had given $\sim 13 \%-15 \%$ upper limits on direct photon production from central collisions. No effect was observed for peripheral collisions. 
Later, $\mathrm{Pb}-\mathrm{Pb}$ interactions at $\sqrt{\mathrm{s}}_{\mathrm{nn}}=17.3 \mathrm{GeV}$ studied by WA98 [3] found an excess at high $\mathrm{p}_{\mathrm{T}}$ for central collisions, not seen in peripheral collisions, as shown in Fig. 1.

A model in the frame of QGP and HG formation [4] describes the results with an initial high temperature of $T_{i}=270 \mathrm{MeV}$. Nevertheless, using a lower temperature, $T_{i}=205 \mathrm{MeV}$ and a $\mathrm{k}_{\mathrm{T}}$ broadening also describes the data.

Fig. 2 shows the results obtained in Au-Au collisions at $\sqrt{ }_{\mathrm{n}_{\mathrm{n}}}=200 \mathrm{GeV}$ by PHENIX collaboration for real photon production per $\pi^{0}$ as a function of $\mathrm{p}_{\mathrm{T}}$ compared with the expected spectrum of background photons coming from hadronic decays [5]. An excess increasing with centrality is observed for $\mathrm{p}_{\mathrm{T}}>4 \mathrm{GeV} / \mathrm{c}$ due to the $\pi^{0}$ suppression. This $\pi^{0}$ suppression was not observed at SPS energies. NLO pQCD calculations scaled with the number of binary nucleonnucleon collisions accounts for the measured photons yield [6].

\subsection{Low Mass Lepton Pairs}

A pioneering result for electron pairs production at low masses (below $\phi$ resonance), was obtained by CERES/SPS experiment in S-Au collisions at $\sqrt{ }_{\mathrm{sn}_{\mathrm{n}}}=19.4 \mathrm{GeV}$ [7]. An enhancement was observed as compared to the expected hadronic sources that describe the results in protoninduced interactions as shown in Fig. 3. Results obtained in $\mathrm{Pb}-\mathrm{Pb}$ collisions at $\sqrt{ }_{\mathrm{sn}_{\mathrm{n}}}=17.3 \mathrm{GeV}$ [8] present also an excess. This excess is interpreted in terms of thermal production by $\pi^{+} \pi^{-}$ annihilation from the dense hadronic gas created in nuclear collisions together with a $\rho$ inmedium modification (a broadening of $\rho$ spectral shape [9] or a $\rho$-mass shift due to chiral symmetry restoration [10]). In Fig. 4 the comparison of those models with data is shown, but data precision is not enough to disentangle between them. The excess was not observed by NA38/50 experiments by studying high $\mathrm{p}_{\mathrm{T}}$ low mass muon pairs production in $\mathrm{p}-\mathrm{U}, \mathrm{S}-\mathrm{Cu}, \mathrm{S}-\mathrm{U}$ and $\mathrm{Pb}-\mathrm{Pb}$ collisions [11]. Latest results in $\mathrm{Pb}-\mathrm{Au}$ interactions from CERES [12] show that the excess increases at low $\mathrm{p}_{\mathrm{T}}$ (a factor $\sim 3$ for $\mathrm{p}_{\mathrm{T}}>200 \mathrm{MeV}$ and a factor $\sim 6$ for $\mathrm{p}_{\mathrm{T}}>100 \mathrm{MeV}$ ).

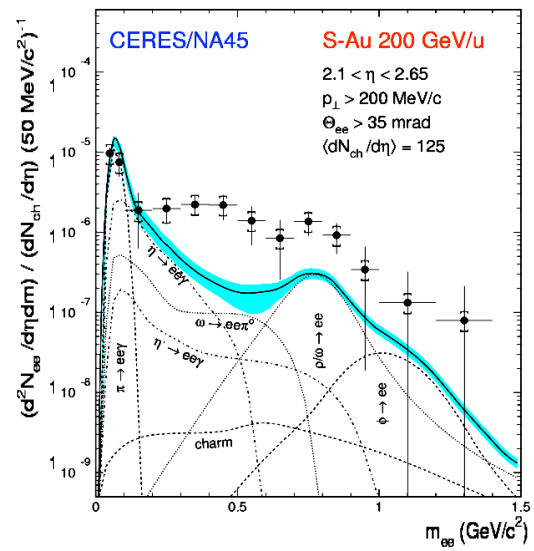

Figure 3. Low mass dielectron distribution for $\mathrm{S}-\mathrm{Au}$ collisions. The overall known sources contribution is represented by the line shaded in cyan.

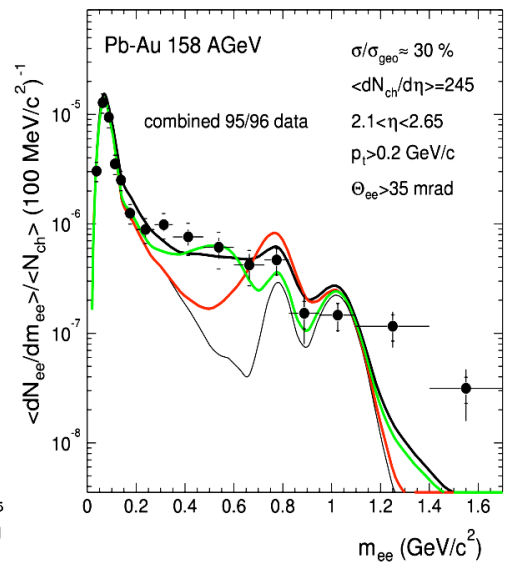

Figure 4. Low mass dielectron distribution measured in $\mathrm{Pb}-\mathrm{Au}$ collisions by CERES. The lines represent models including or not $\rho$ thermal production.

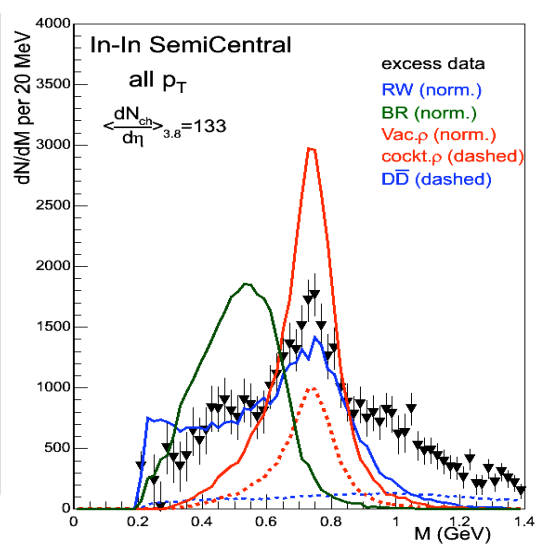

Figure 5. The excess as a function of dimuon mass measured in semi-central In-In collisions measured by NA60 and compared with models. 
Recently, NA60/SPS experiment, using the NA38/NA50 dimuon spectrometer with improved mass resolution and better low mass and low $\mathrm{p}_{\mathrm{T}}$ acceptances showed that whereas proton induced interactions can be described as a superposition of known sources an excess is observed in In-In collisions at $\sqrt{ }_{\mathrm{s}_{\mathrm{nn}}}=17.3 \mathrm{GeV}$ [13]. In Fig. 5, the mass distribution of this excess is compared with two models that include a $\rho$ in-medium modification: a broadening of the $\rho$ spectral shape (blue line [9]) which is in agreement with data and a shift of the $\rho$ pole (green line [10]), which is clearly ruled out.

\subsection{Virtual Photons and Heavy Quarks}

A signal of thermal virtual photon emission by the thermalized QGP or hadron gas created in ultra-relativistic nuclear collisions is expected to be seen in the di-lepton mass spectrum between the $\phi$ and the $J / \psi$ mass resonances, the so-called intermediate mass region (IMR). Indeed, in Helios/3 [14], NA38 and NA50 [15] experiments an excess was observed compared to the expected sources - Drell-Yan and open charm production scaled linearly with the mass number.

The excess measured by NA50 in Pb-Pb central collisions is well described as an open charm enhancement (a factor of 3.5 is measured for the most central collisions) or as a thermal radiation from QGP phase according to theoretical calculations [16] as shown in Fig. 6.

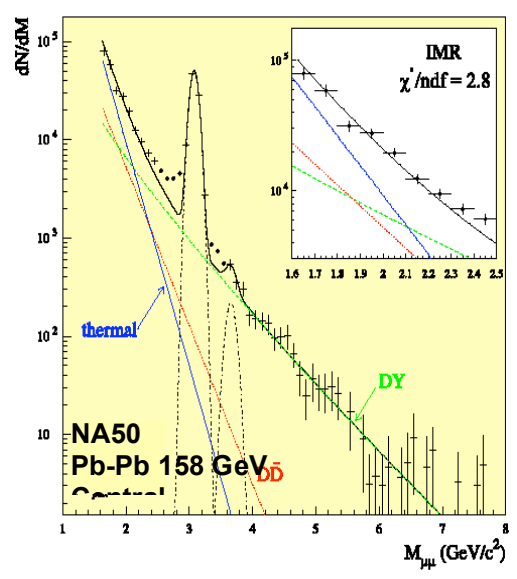

Figure 6. Dimuon mass distribution measured in $\mathrm{Pb}-\mathrm{Pb}$ central collisions by NA50. The IMR fit includes expected DY and open charm sources and thermal dimuon production.

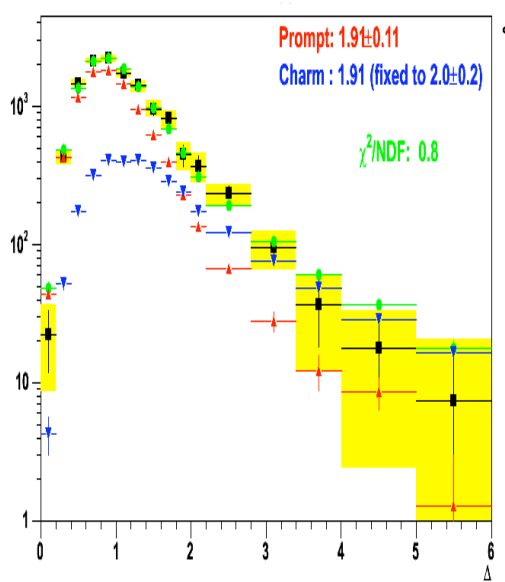

Figure 7. Fit of NA60 In-In IMR dimuon weighted offset spectrum with fixed open charm and free prompt contribution.

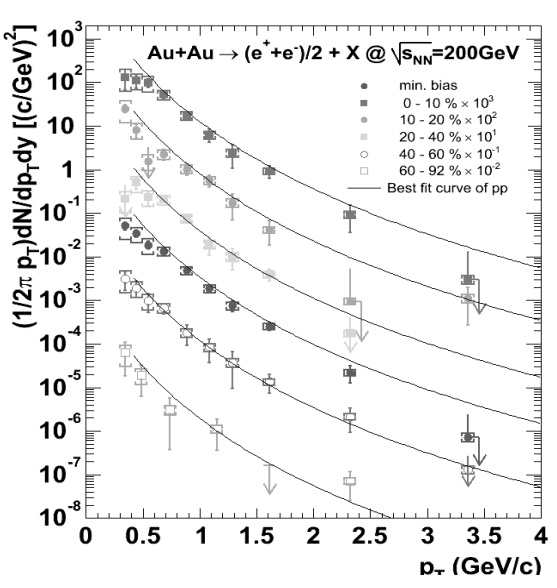

Figure 8. Non-photonic $\mathrm{e}^{+}+\mathrm{e}^{-} \mathrm{p}_{\mathrm{T}}$ sepectra for $\mathrm{Au}-\mathrm{Au}$ collisions measured by PHENIX, for different centralities. The curves represent the best fit for $p p$ data, binary rescaled.

The vertex resolution obtained in NA60 experiment allows to distinguish between prompt dimuon production and the muons coming from $\mathrm{D}$ meson semi-leptonic decays by identifying its decay vertex. At this conference, a distribution of the weighted muons offsets with respect to the position of the primary vertex was shown and compared to the expected offset distributions 
for prompt and decay muons [17]. In Fig. 7, the fit result is shown considering the charm contribution fixed to the NA50 p-A expected value $\left(\sigma_{\mathrm{cc}}=12 \mu \mathrm{b} /\right.$ nucleon $)$ and a free contribution for prompt dimuons. The observed excess (almost a factor of 2 ) is compatible with a prompt source.

At PHENIX [18] and STAR [19] experiments, charm decay signal is obtained by the nonphotonic single $\mathrm{e}^{+}+\mathrm{e}^{-}$spectra, subtracted for $\gamma$ conversions of decays from $\pi^{0}$ and $\eta$ hadrons and Dalitz pairs. In Au-Au collisions, the data compared with NLO QCD calculations, rescaled by the number of binary collisions from the pp reference, show no enhancement or any effect as a function of centrality (see Fig. 8), but systematic errors are important.

\section{Hard Probes}

Penetrating probes, as quarkonia production and hard scattering, are very relevant for the study of the early stages of QGP formation, namely the pre-equilibrium and the deconfinement. Besides, they also probe the medium density.

\subsection{Quarkonia Dissociation}

Matsui and Satz predicted that in a deconfined medium heavy quarkonium states (charmonia and bottonia) are prevented to form due to Debye colour screening [20].

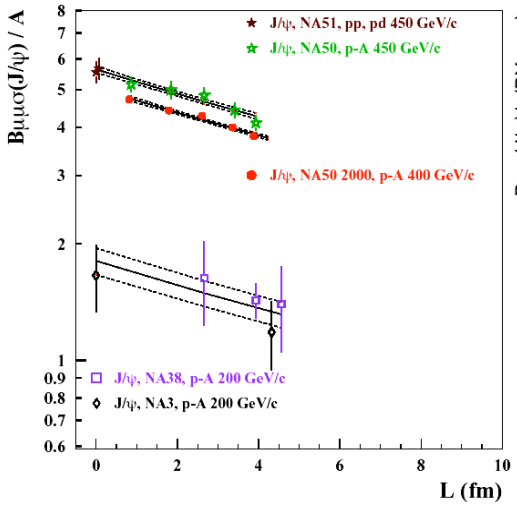

Figure 9. $\mathrm{J} / \psi$ cross-section per nucleon as a function of $\mathrm{L}$ Results obtained at SPS experiments and fitted with Glauber model.

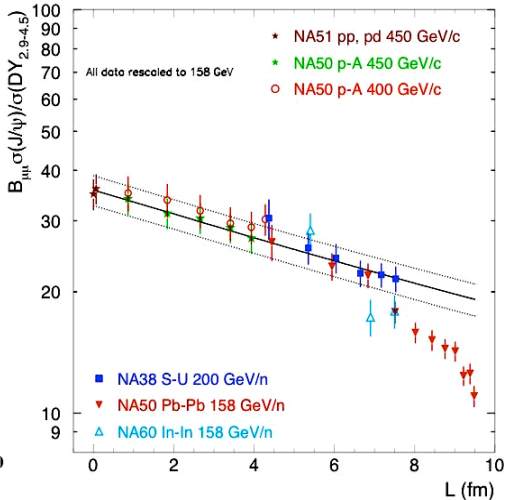

Figure 10. Results on the crosssection ratio $(\mathrm{J} / \psi) / \mathrm{DY}$ vs $\mathrm{L}$, measured in $\mathrm{pp}$ collisions up to $\mathrm{Pb}-\mathrm{Pb}$, rescaled to $158 \mathrm{GeV}$.

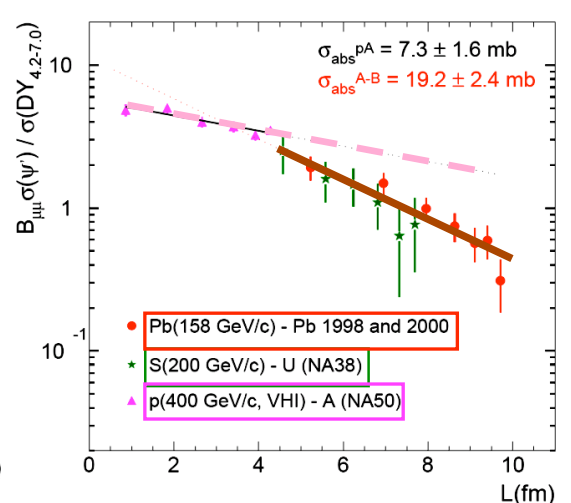

Figure 11. Results on the crosssection ratio $\psi^{\prime} / \mathrm{DY}$ vs $\mathrm{L}$, measured in $\mathrm{pp}$ and $\mathrm{S}-\mathrm{U}$, rescaled to $158 \mathrm{GeV}$, and $\mathrm{Pb}-\mathrm{Pb}$ collisions. Lines represents exponential fits.

NA50 experiment observed an anomalous $\mathrm{J} / \psi$ suppression in $\mathrm{Pb}-\mathrm{Pb}$ collisions with $\mathrm{a}$ certain centrality [21]. This effect can not be simply described as an hadronic absorption. In fact, NA50 made recently a complete and coherent analysis of $\mathrm{J} / \psi$ and $\psi^{\prime}$ produced in proton induced interactions, in order to establish the baseline of charmonia production [22]. Fig. 9 shows the $\mathrm{J} / \psi$ cross-section per nucleon as a function of $\mathrm{L}$ (the mean free path traversed by the pre-resonance in nuclear matter), obtained by NA3/38/50/51 (all experiments, except the first, 
use the same dimuon spectrometer). Proton-nucleus data are fitted with Glauber model giving the absorption cross-section $\sigma_{\text {abs }}(\mathrm{J} / \psi)=4.48 \pm 0.42 \mathrm{mb}$. Using this fit result and rescaling all data to the $\mathrm{Pb}-\mathrm{Pb}$ energy, a clear $\mathrm{J} / \psi$ anomalous suppression is observed for semi-central and central $\mathrm{Pb}-\mathrm{Pb}$ collisions which increases with centrality [23], as shown in Fig. 10. In this figure it is also seen that the results measured in S-U interactions agree with the reference curve, leaving very little room for other suppression mechanism. The recent preliminary In-In NA60 results present also an anomalous suppression in central collisions [24], but the L variable seems not to be the good one to describe both $\mathrm{J} / \psi$ suppressions obtained in the two systems, $\mathrm{Pb}-\mathrm{Pb}$ and $\mathrm{In}-\mathrm{In}$.

The $\psi$ ' resonance is much more suppressed in ion collisions then $\mathrm{J} / \psi$. Also the $\psi$ ' has an important nuclear absorption: $\sigma_{\mathrm{abs}}\left(\psi^{\prime}\right)=7.3 \pm 1.6 \mathrm{mb}$, value measured by NA50 in protonnucleus collisions [22]. Both proton and ion results on $\psi$ ' production as a function of L, normalized to the DY reference, are shown in Fig. 11. The simultaneous fit result of $\mathrm{B}_{\mu \mu} \sigma\left(\psi^{\prime}\right) / \sigma(\mathrm{DY})$ obtained in $\mathrm{S}-\mathrm{U}$ and $\mathrm{Pb}-\mathrm{Pb}$ collisions (recent and coherent data analyses), using the exponential parameterisation $\sigma_{0} \mathrm{e}^{-<\rho L>\text { oabs }}$, lead to a huge value for $\sigma_{\mathrm{abs}}\left(\psi^{\prime}\right)=19.2 \pm 2.4 \mathrm{mb}$ [25].

The SPS results obtained for charmonia anomalous suppression are summarized in Fig. 12 in terms of a ratio dependence on the number of participants $\left(\mathrm{N}_{\text {part }}\right)$ of the measured $\mathrm{J} / \psi$ (or $\psi^{\prime}$ ) yield, normalized by the Drell-Yan events, over the expected yield assuming only normal nuclear absorption as measured in p-A collisions. The $\psi$ ' anomalous suppression sets in at lower centralities as $J / \psi$ and it is already observed in S-U interactions. This difference between $J / \psi$ and $\psi$ ' patterns could be due to the lower temperature achieved in S-U collisions which only would prevent the formation of the less bound $\psi^{\prime}$ state. The In-In results for $J / \psi$ are in agreement with the suppression measured in $\mathrm{Pb}-\mathrm{Pb}$ when $\mathrm{N}_{\text {part }}$ is used.

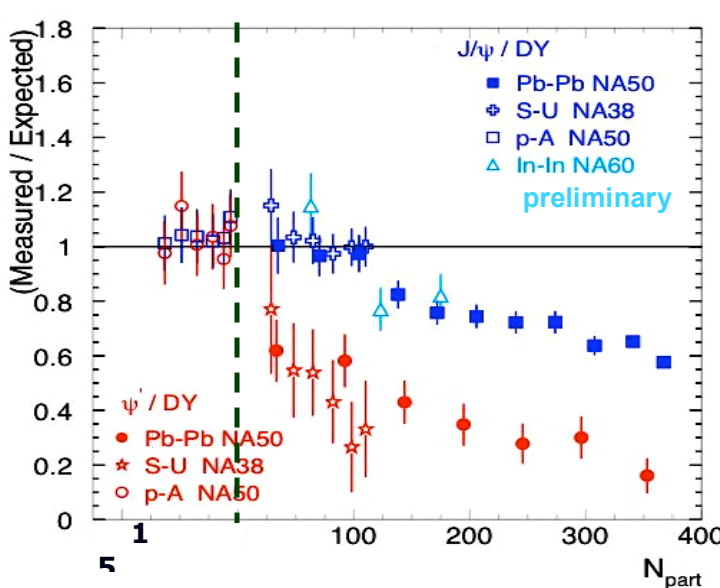

Figure 12. $\mathrm{J} / \psi$ and $\psi^{\prime}$ 'suppression pattern as a function of $\mathrm{N}_{\text {part }}$ for several collisions at SPS.

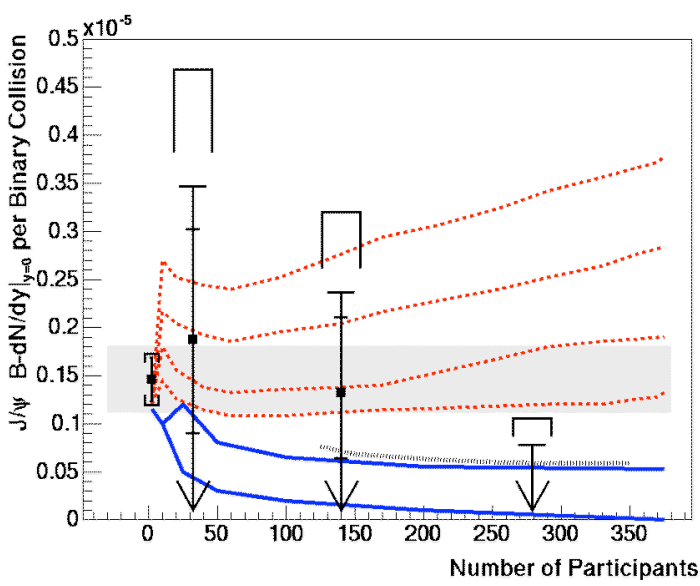

Figure 13. PHENIX results on the $J / \psi$ production per binary collision as a function of $\mathrm{N}_{\text {part }}$.

No new results from RHIC were presented at this conference and only the result of the $J / \psi$ yield per binary collision as a function of $\mathrm{N}_{\text {part }}$ corresponding to a very low statistics sample was shown, see Fig. 13 [26]. The models predicting at RHIC energies strong enhancement are ruled 
out, being the results more consistent with the suppression observed at SPS energies, although results with higher statistics are desirable.

So far, the existing models giving $J / \psi$ suppression can be better tested with results obtained with ion collisions at SPS. In Figs. 14-16, the J/ $\psi$ pattern suppression as a function of $\mathrm{N}_{\text {part }}$ is compared with three different models. A model [27] considering $\mathrm{J} / \psi$ absorption by comover hadrons tuned to $\mathrm{S}-\mathrm{U}$ and $\mathrm{Pb}-\mathrm{Pb}$ data is extrapolated to In-In. The model does not describe the normal $\mathrm{J} / \psi$ nuclear absorption as seen in Fig. 14 in terms of the ratio between $\mathrm{J} / \psi$ measured in p-A and the model result (dashed dot line) and also fails its prediction for $\mathrm{J} / \psi$ suppression in In-In (dotted line). A second model [28] assuming heavy quarkonia suppression in a deconfined medium within percolation scenario describes $\mathrm{Pb}-\mathrm{Pb} \mathrm{J} / \psi$ pattern (orange curve of Fig. 15), but fails its prediction for In-In (magenta curve). And a third model [29] in the frame of QGP formation and including thermal regeneration seems to describe both ion system results (violet curve of Fig. 16). Nevertheless, there is some discrepancy in the In-In results at high centralities, which needs to be investigated.

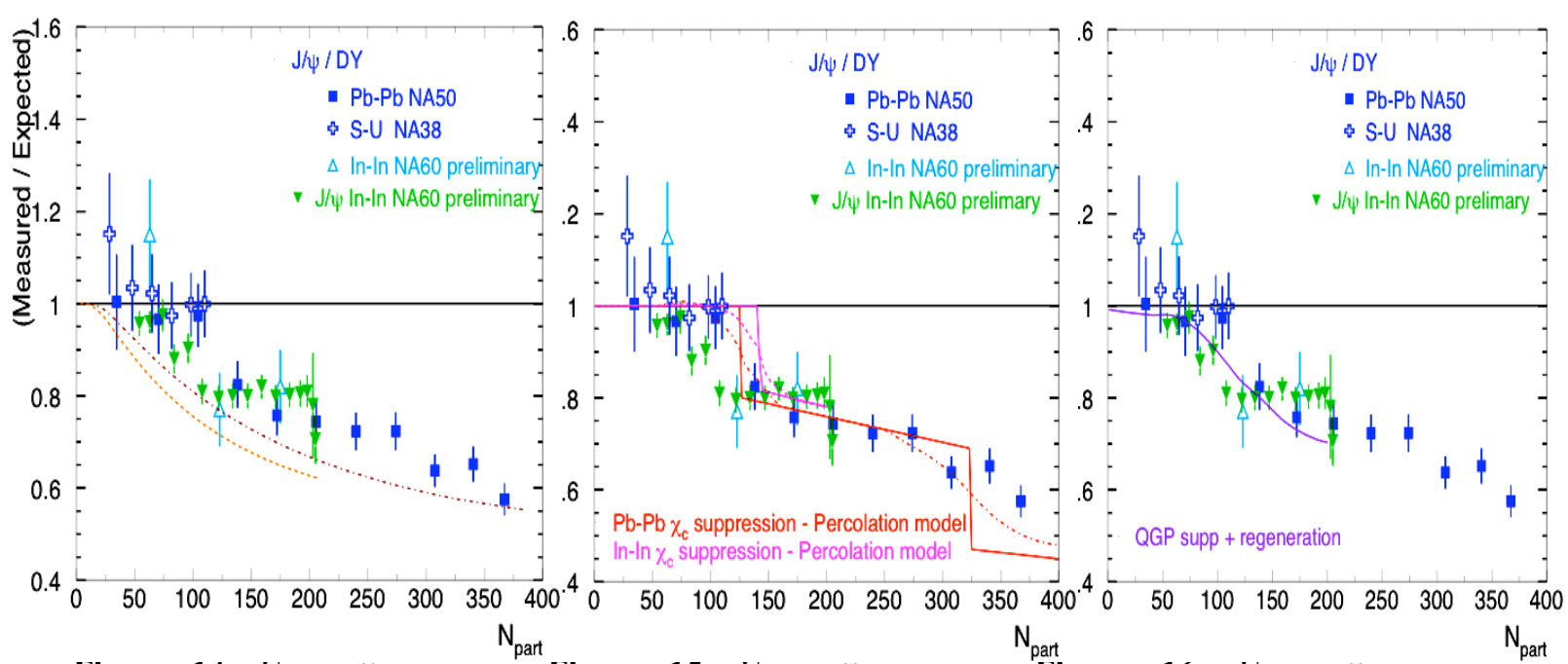

Figure 14. $\mathrm{J} / \psi$ pattern as a Figure 15. $\mathrm{J} / \psi$ pattern as a Figure 16. $\mathrm{J} / \psi$ pattern as a function of $\mathrm{N}_{\text {part }}$ compared with function of $\mathrm{N}_{\text {part }}$ compared with a a comover model. percolation model.

function of $\mathrm{N}_{\text {part }}$ compared with a QGP model with thermal regeneration.

First results on $\Upsilon$ production in proton-nucleus collisions were presented by NA50 collaboration [cortese]. The mass fit considers $\Upsilon$ states and Drell-Yan dimuons and the results gives $\Upsilon$ scaling as $A^{\alpha}$, with $\alpha=0.98 \pm 0.08$, that is, compatible with 1 , meaning that $\Upsilon$ is weakly absorbed by nuclear matter, see Fig. 17. The measurement of $\Upsilon$ production is very important in view of LHC where the $\Upsilon$ dissociation in $\mathrm{Pb}-\mathrm{Pb}$ collisions is expected to occur.

In Fig. 18 a compilation on $\Upsilon$ cross-section measurements at mid-rapidity is made as a function of $\sqrt{ }$ s. Assuming $\alpha_{Y}=1$, a very good agreement with colour evaporated model NLO calculations (CEM) is seen [30]. 


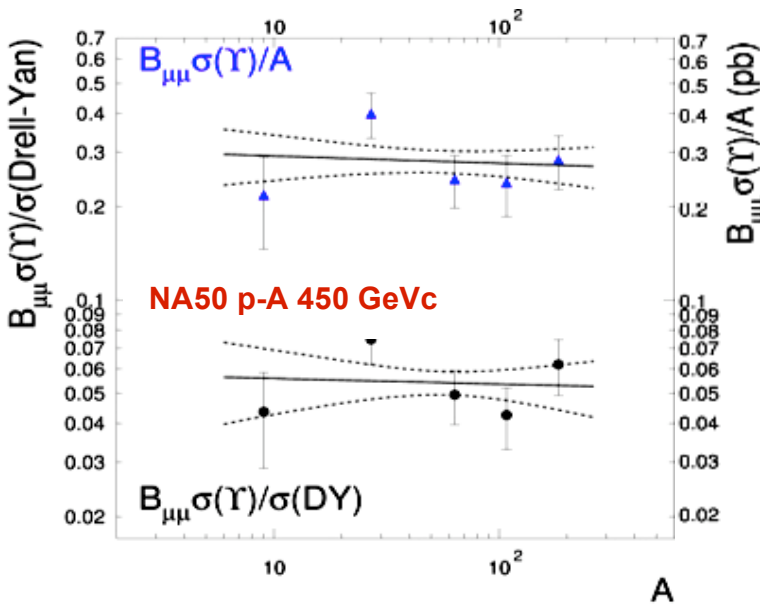

Figure 17. $\Upsilon$ cross-section per nucleon-nucleon collision and the cross-section ratio $B_{\mu \mu} \sigma_{Y} / \sigma_{D Y}$ measured in $\mathrm{p}$-A collisions as a function of $\mathrm{A}$.

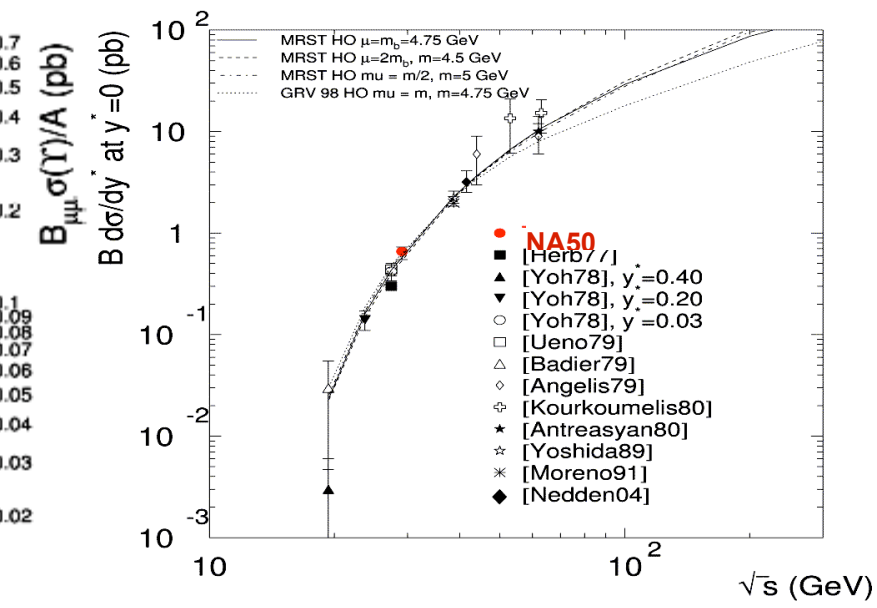

Figure 18. $\Upsilon$ cross-section at mid-rapidity as a function of $\sqrt{\mathrm{s}}$, compared with CEM calculations.

\subsection{Jet Quenching}

The suppression of the yield of high $\mathrm{p}_{\mathrm{T}}$ hadrons produced in ultra-relativistic nucleusnucleus collisions relative to the yield extrapolated from pp interactions [31] has become one of the relevant probes of strongly interacting matter creation. As shown in Fig. 19, the four RHIC experiments measure a suppression factor up to 5 in the most central collisions in terms of nuclear modification factor defined as: $R_{A A}=d^{2} N / d p_{T} d \eta /\left(\left(<N_{b i n}>/ \sigma_{\text {inel }}{ }^{N N}\right) d^{2} \sigma^{N N} / d p_{T} d \eta\right)$.

It was predicted that energy loss of high momentum partons via induced gluon radiation would occur in a hot and dense medium created at early stages of the ultra-relativistic nucleusnucleus collisions [32]. It is the so-called jet quenching.

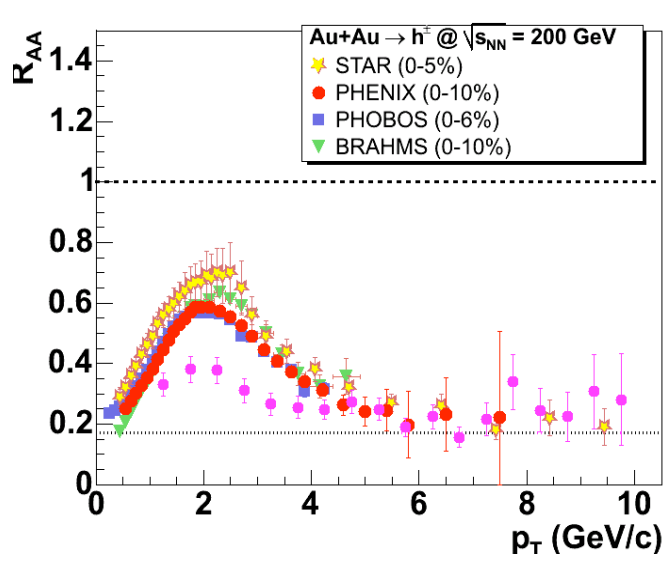

Figure 19. Nuclear modification factors for charged hadrons produced in central $\mathrm{Au}-\mathrm{Au}$ collisions as a function of $\mathrm{A}$. The magenta doted points correspond to the $\pi^{0}$ production.

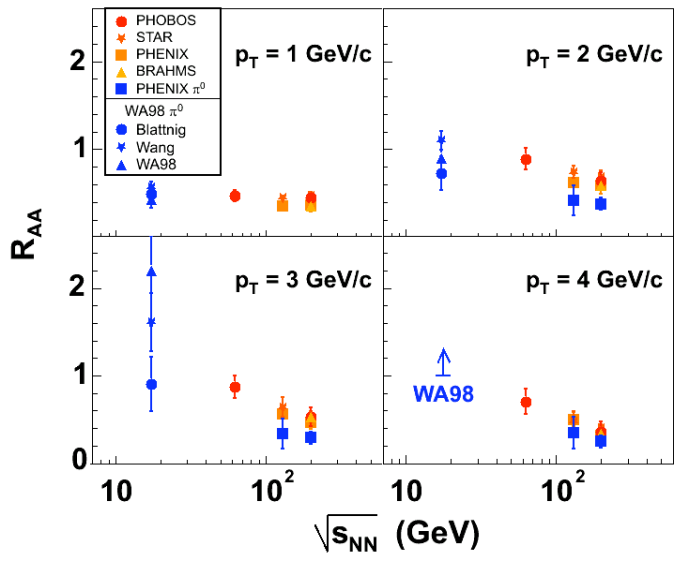

Figure 20. $\mathrm{R}_{\mathrm{AA}}$ as a function of $\sqrt{\mathrm{s}}$ for the $6 \%$ most central collisions and for different $p_{T}$ classes. $\pi^{0}$ data is also shown (blue points).

To disentangle between two possible interpretations of these results - final state effects due to parton energy loss in a dense medium or initial state effects, the RHIC experiments 
studied d-Au collisions. In these reactions, initial state events are still expected to be present contrary to final state effects as no dense matter is produced. From the results [33-36] shown in Fig. 21 at mid-rapidity, one observes that the charged hadron yield exceeds the binary scaling expectation. This rules out initial state effects, being the enhancement due to the Cronin effect. This leads to the conclusion that the observed high $\mathrm{p}_{\mathrm{T}}$ suppression in Au-Au collisions is related to the presence of a dense and hot medium.
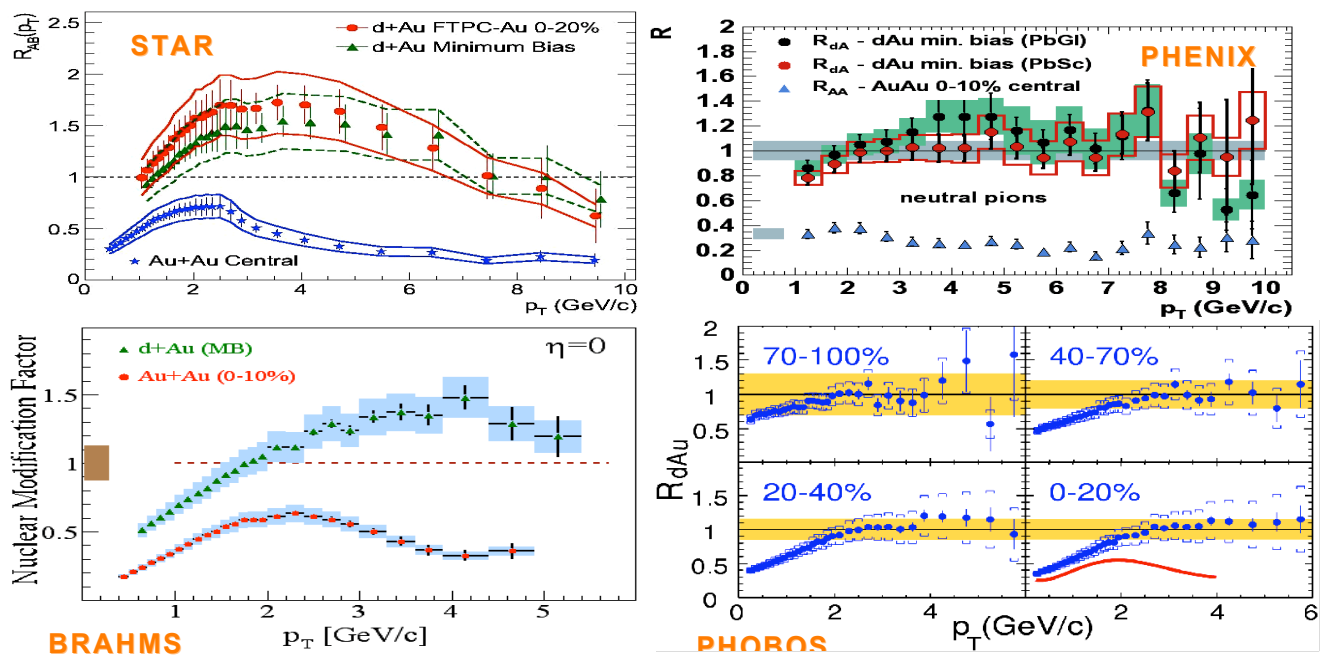

Figure 21. Nuclear modification factor as a function of $\mathrm{p}_{\mathrm{T}}$ measured in d-Au and $\mathrm{Au}-\mathrm{Au}$ mid-rapidity collisions by the four RHIC experiments.

One also observes that the high $\mathrm{p}_{\mathrm{T}}$ suppression increases with centrality and, as shown in Fig. 20, $\mathrm{R}_{\mathrm{AA}}$ decreases with $\sqrt{\mathrm{s}}$ for the $6 \%$ most central $\mathrm{Au}$-Au collisions [37].
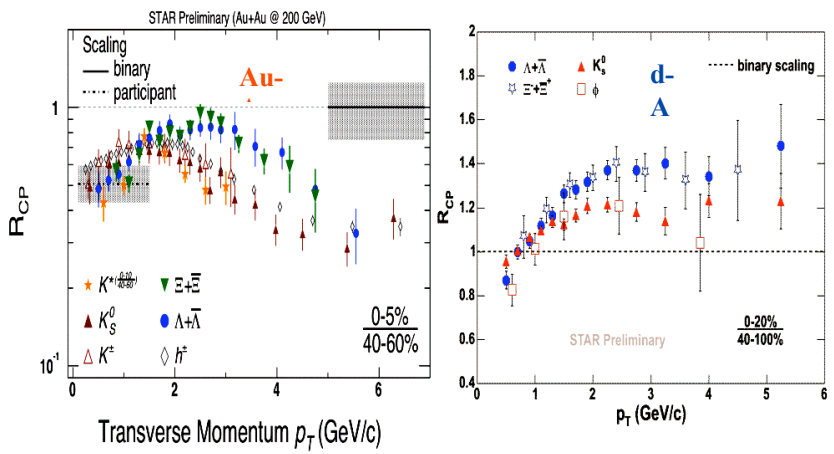

Figure 22. $\mathrm{R}_{\mathrm{CP}}$ factor as a function of $\mathrm{p}_{\mathrm{T}}$ measured in $\mathrm{Au}-\mathrm{Au}$ (left plot) and d-Au collisions (right plot) by STAR experiment for baryons and mesons.
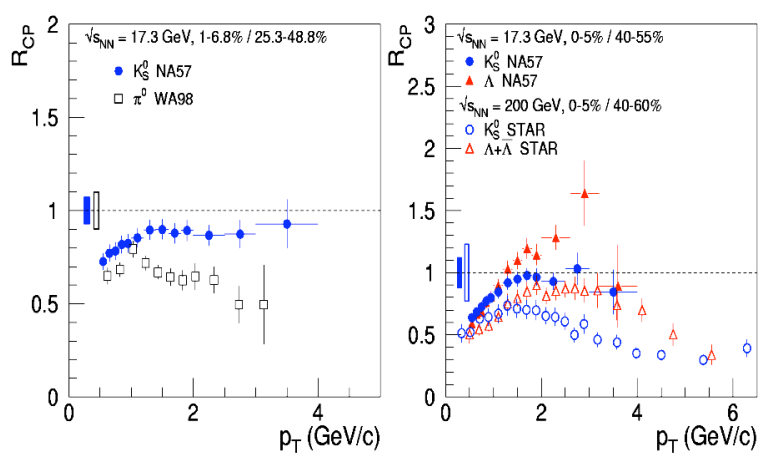

Figure 23. Left plot: $\mathrm{R}_{\mathrm{CP}}$ for $\mathrm{K}_{\mathrm{S}}^{0}$ and $\pi^{0}$ in $\mathrm{Pb}$ $\mathrm{Pb}$ collisions at SPS. Right plot: $\mathrm{R}_{\mathrm{CP}}$ for $\mathrm{K}_{\mathrm{S}}{ }^{0}$ and $\Lambda$ measured by NA57 and STAR experiments.

The high $\mathrm{p}_{\mathrm{T}}$ suppression can also be studied in terms of a $\mathrm{R}_{\mathrm{CP}}$ factor. $\mathrm{R}_{\mathrm{CP}}$ is defined as the $\mathrm{p}_{\mathrm{T}}$ spectra ratio for central and peripheral collisions, normalized by the corresponding number of binary collisions: $\mathrm{R}_{\mathrm{CP}}=\left(<\mathrm{N}_{\text {coll }}>_{\mathrm{P}} /<\mathrm{N}_{\text {coll }}>_{\mathrm{C}}\right) \cdot\left(\mathrm{d}^{2} \mathrm{~N}_{\mathrm{C}} / \mathrm{dp}_{\mathrm{T}} \mathrm{dy}\right) /\left(\mathrm{d}^{2} \mathrm{~N}_{\mathrm{P}} / \mathrm{dp}_{\mathrm{T}} \mathrm{dy}\right)$. The $\mathrm{R}_{\mathrm{CP}}$ results from STAR experiment at $\sqrt{S}=200 \mathrm{GeV}$ are shown in Fig. 22 for different particle species [38]. It is found that mesons are more suppressed than baryons in both Au and d induced 
collisions. At SPS energies, this effect was also observed by NA57/WA98 in Pb-Pb collisions [39], but with higher $R_{C P}$ values, cf. Fig. 23. The similar K- $\Lambda$ pattern observed at both energies may indicate baryon/meson recombination effect, but a Cronin effect larger for $\Lambda$ with respect to $\mathrm{K}$ cannot be discarded.
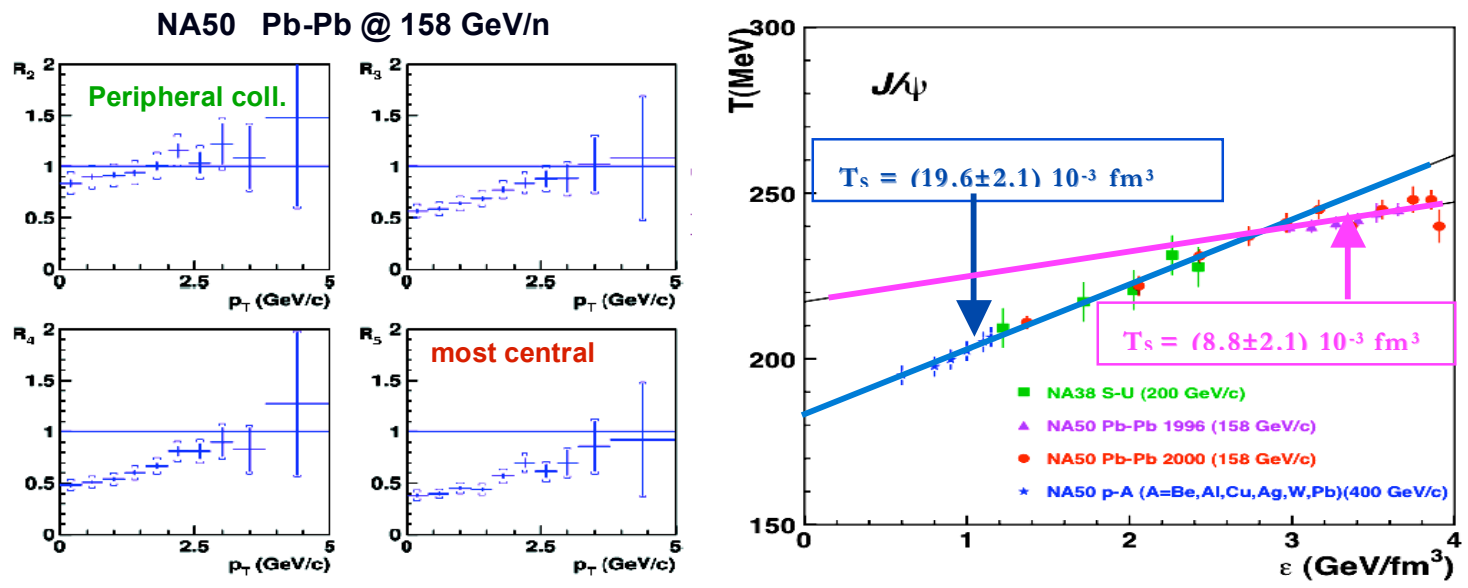

Figure 24. $\mathrm{R}_{\mathrm{CP}}$ factor for $\mathrm{J} / \psi$ as a function of $\mathrm{p}_{\mathrm{T}}$ and different centrality classes, measured in $\mathrm{Pb}-\mathrm{Pb}$ collisions at $\sqrt{\mathrm{s}}=17.3 \mathrm{GeV}$ by NA50 experiment.

Figure 25. $\mathrm{T}$ for $\mathrm{J} / \psi$ as a function of $\varepsilon$. pA and $\mathrm{S}$ $\mathrm{U}$ data are rescaled to $\sqrt{\mathrm{s}}_{\mathrm{s}}=17.3 \mathrm{GeV}$. Linear fits give a lower slope $\left(\mathrm{T}_{\mathrm{S}}\right)$ for central collisions .

Similar behaviour is observed for $\mathrm{J} / \psi$ mesons: the nuclear modification factor as measured by NA50 experiment in the most central $\mathrm{Pb}-\mathrm{Pb}$ collisions (here the normalization is defined from the measured DY spectrum) [40] shows a saturation (below 1) as displayed in Fig. 24. This behaviour is contrary to the one obtained in S-U collisions by NA38, where a $k_{T}$ broadening is seen, leading to $\mathrm{R}_{\mathrm{CP}}\left(\mathrm{p}_{\mathrm{T}}\right)>1$, also found in peripheral $\mathrm{Pb}-\mathrm{Pb}$ collisions.

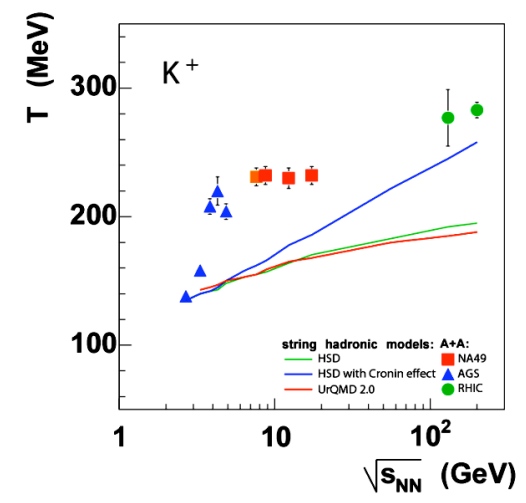

Figure 26. Energy dependence of $\mathrm{T}$ for $\mathrm{K}^{+}$produced in central collisions at AGS, SPS and RHIC. The lines are hadronic models.

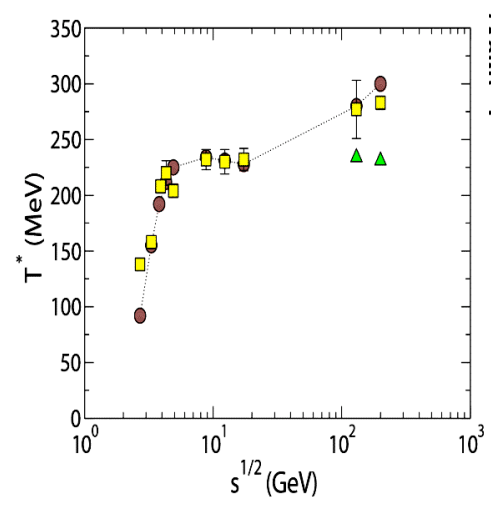

Figure 27. Same data as Fig 26 compared with a hydrodynamic model (brown points).

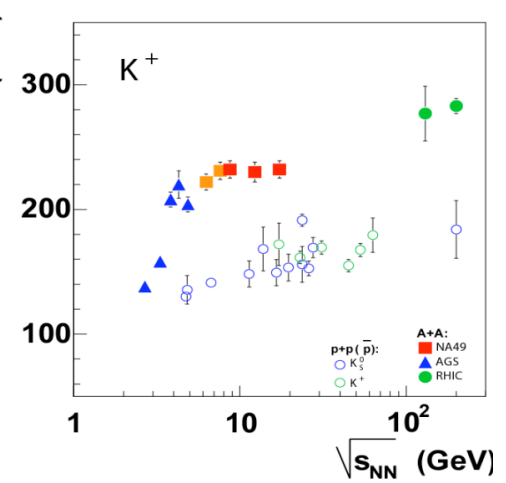

Figure 28. Energy dependence of $\mathrm{T}$ for $\mathrm{K}$ mesons produced in central A-A collisions and in pp collisions.

NA49 made a systematic study of hadron production as a function of $\sqrt{s}$ [41]. From transverse mass fits of $\mathrm{K}^{+}$produced at mid-rapidity and central collisions, the inverse slope parameter T is extracted and compared with AGS and RHIC results, plotted in Fig. 26. A step- 
like behaviour that is not explained by transport models [42] which use hadronic and string degrees of freedom is found, but it is consistent with a modification of the equation of state from confined to deconfined matter, as predicted by [43]. Calculations with a hydrodynamic model, assuming a first order phase transition to QGP [44] describes the data as seen in Fig. 27. This behaviour is not present in pp collisions, displayed in Fig. 28.

A similar effect is observed in terms of the energy density dependence of the $T$ values measured for $\mathrm{J} / \psi$ by NA50/38 experiments, cf. Fig. 25 [45]. Data shows a flattening behaviour of $\mathrm{T}$ for $\mathrm{J} / \psi$ produced in $\mathrm{Pb}-\mathrm{Pb}$ central collisions, as contrary to the rise observed in peripheral $\mathrm{Pb}-\mathrm{Pb}, \mathrm{S}-\mathrm{U}$ and $\mathrm{pp}$ collisions. This is expressed in terms of a slope parameter $\mathrm{T}_{\mathrm{S}}$ obtained from a linear fit, giving $T_{\mathrm{S}}=(8.8 \pm 2.1) 10^{-3} \mathrm{fm}^{3}$ (central) and $\mathrm{T}_{\mathrm{S}}=(8.8 \pm 2.1) 10^{-3} \mathrm{fm}^{3}$ (peripheral).

Finally, STAR experiment found a strong suppression of back-to-back high $\mathrm{p}_{\mathrm{T}}$ hadron pairs angular correlation (jet-like correlations) in Au-Au central collisions at $\mathrm{s}=200 \mathrm{GeV}$ [46]. In Fig 29, a comparison of the relative azimuthal angle distribution $(\Delta \phi)$ of high $\mathrm{p}_{\mathrm{T}}$ di-hadrons produced in central $\mathrm{Au}-\mathrm{Au}$ collisions to those produced in $\mathrm{pp}$ and $\mathrm{d}-\mathrm{Au}$ central collisions shows different behaviour for back-to-back pairs while similar for near-side pairs. This is consistent within a jet quenching scenario: the leading fragments of the recoiling jet are suppressed due to medium-induced partonic energy loss. Moreover, the study of $\Delta \phi$ in non-central $\mathrm{Au}-\mathrm{Au}$ collisions with respect to the reaction plane exhibits a larger suppression in the back-to-back distribution for out-of-plane triggers [47], as shown in Fig. 30. This means that for out-of-plane triggers, the partons lose more energy as their path length on traversing the system becomes larger.

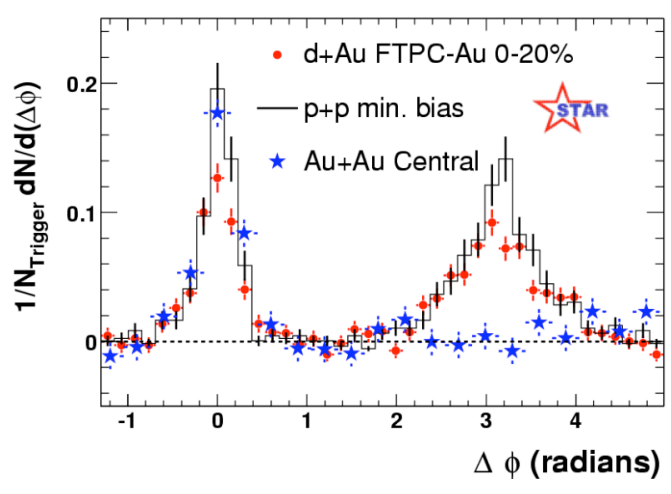

Figure 29. Relative azimuthal angle distributions for high $\mathrm{p}_{\mathrm{T}}$ di-hadrons measured in $\mathrm{pp}, \mathrm{d}-\mathrm{Au}$ and $\mathrm{Au}-\mathrm{Au}$ central collisions at $\sqrt{\mathrm{s}}=200 \mathrm{GeV}$ by STAR.

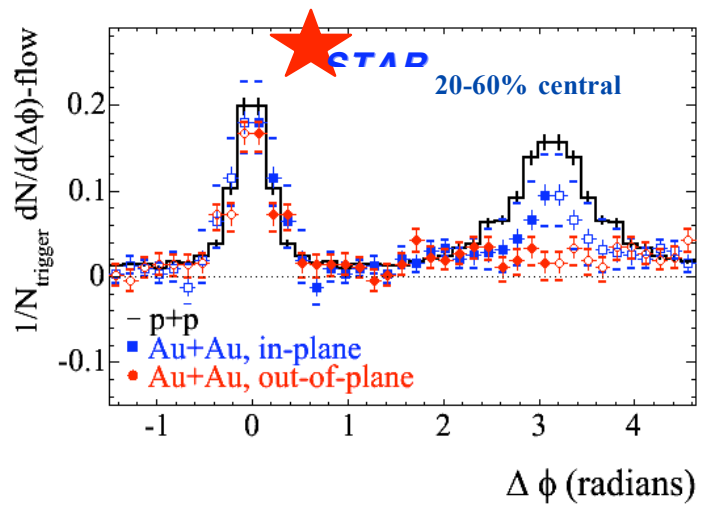

Figure 30. Two-particle azimuthal distributions for trigger particles in or out of reaction plane in mid-central $\mathrm{Au}-\mathrm{Au}$ collisions and for $\mathrm{pp}$.

In conclusion, an impressive amount of interesting results with anomalous behaviour were obtained in ultra-relativistic heavy ion collisions at SPS/CERN and RHIC/BNL, pointing to the formation of a new state of matter. A bright future is thus foreseen at RHIC and LHC.

\section{References}

[1] F. Karsh, Nucl. Phys. A 698 (2002) 199; P. Petreczky, Nul. Phys. B 140 (2005) 78. 
[2] R. Albrecht et al. (WA80 Coll.) Z. Phys. C 51 (1991) 1; P.C. Bosetti et al. (HELIOS/2 Coll.), Z. Phys. C 46 (1990) 377; R. Bauer et al. (CERES Coll.), Z. Phys. C 71 (1996) 571.

[3] M.M. Agarwal et al. (WA98 Coll.), Phys. Rev. Lett. 85 (2000) 3595.

[4] S. Turbide et al., Phys. Rev. C 69 (2004) 014902.

[5] S.S. Adler et al. (PHENIX Coll.), Phys. Rev. Lett. 94 (2004) 232301.

[6] B. Jager et al., Phys. Rev. D 67, (2003) 054005.

[7] G. Agakichiev et al. (CERES Coll.) Phys. Rev. Lett. 75 (1995) 1272.

[8] G. Agakichiev et al. (CERES coll.) Phys. Lett. B 422 (1998) 405, Agakichiev et al. (CERES Coll.) Nucl.Phys. A 661 (1999) 23.

[9] R. Rapp and J. Wambach, Adv. Nucl. Phys. 25 (2000) 1.

[10] G. E. Brown and M. Rho, Phys. Rept. 363 (2002) 85.

[11] M.C. Abreu et al. (NA38/50 Coll.) Eur. Phys. J. C 13 (2000) 69.

[12] G. Agakichiev et al. (CERES Coll.) Eur. Phys. J. C 41 (2005) 475.

[13]H.K. Whori et al. (NA60 Coll.), these proceedings.

[14] A.L.S. Angelis et al. (HELIOS/3 Coll.) Eur. Phys. J. C 13 (2000) 433.

[15] M.C. Abreu et al. (NA38/50 Coll.) Eur. Phys. J. C 14 (2000) 443.

[16]R. Rapp and E. Shuryak, Phys. Lett. B 473 (2000) 13.

[17] R. Shahoyan et al. (NA60 Coll.), these proceedings.

[18] S.S. Adler et al. (PHENIX Coll.), Phys. Rev. Lett. 94 (2005) 082301.

[19] J. Adams et al. (STAR Coll.), Phys. Rev. Lett. 94 (2005), 062301.

[20] T. Matsui and H. Satz, Phys. Rev. Lett. B 179 (1986) 416.

[21] M.C. Abreu et al. (NA38/50 Coll.) Phys. Lett. B 410 (1997) 337; M.C. Abreu et al. (NA38/50 Coll.), Phys. Lett. B 450 (1999) 337; M.C. Abreu et al. (NA38/50 Coll.), Phys. Lett. B 477 (2000) 337.

[22] G. Borges et al. (NA50 Coll.), J. Phys. G: Nucl. Part. Phys. 30 (2004) S1351; G. Borges et al. (NA50 Coll.), Eur. Phy. J C 43 (2005) 161.

[23] B. Alessandro et al. (NA50 Coll.), Eur. Phys. J. C 39 (2005) 335.

[24] C. Lourenço et al. (NA60 Coll.), these proceedings.

[25]H. Santos et al. (NA50 Coll.), J. Phys. G: Nucl. Part. Phys. 30 (2004) S1175.

[26] D. Peressounko et al (PHENIX Coll.), these proceedings; S.S. Adler et al. (PHENIX Coll.), Phys. Rev. C 69 (2004) 014901.

[27] A. Capella and E. Ferreiro, hep-ph/0505032 ; A. Capella and Sousa, nucl-th/0303055.

[28] S. Digal et al., Eur. Phys. J. C 32 (2004) 547.

[29]L. Grandchamp et al., J. Phys. G 30 (2004) S1355.

[30] R. Vogt, Phys. Rep. 310 (1999) 197; Heavy Ion Phys. (2003) 11.

[31] K. Adcox et al. (PHENIX Coll.), Phys. Rev. Lett. 88 (2002) 022301.

[32] M. Gyulassi and M. Plumer, Phys. Lett. B 243, (1990) 432.

[33] B.B. Back et al. (PHOBOS Coll.), Phys. Rev. Lett. 91 (2003) 072302

[34] S.S. Adler et al. (PHENIX Coll.), Phys. Rev. Lett. 91 (2003) 072303.

[35] J. Adams et al. (STAR Coll.), Phys. Rev. Lett. 91 (2003) 072304.

[36] I. Arsene et al. (BRAHMS Coll.), Phys. Rev. Lett. 91 (2003) 072305.

[37] G. Roland et al., Eur. Phys. J. C 39 (2005) 281.

[38] M.A.C. Lamont (STAR Coll.), J. Phys. G: Nucl. Part. Phys. 30 (2004) S963; L. Barnby (STAR Coll.), J. Phys. G: Nucl. Part. Phys. 30 (2004) S1121.

[39] F. Antinori et al. (NA57 Coll.), Phys. Let. B 623 (2005) 17.

[40] P. Bordalo et al. (NA50 Coll.) Nucl. Phys. A 698 (2002) 137c.

[41] S.V. Afanasiev et al., (NA49 Coll.), Phys. Rev. C 66 (2002) 054902; M. Gazdzicki et al. (NA49 Coll.), J. Phys. G: Nucl. Part. Phys. 30 (2004) S701.

[42] E.L. Bratkovskaya et al., Phys. Rev. C 69 (2004) 054907.

[43] L. Van Hoven, Phys. Lett. B 118, (1982) 138.

[44] Y. Hama et al., Braz. J. Phys. 34 (2004) 322.

[45] N.S.Topilskaya et al. (NA50 Coll.), Nucl. Phys. A 715 (2003) 675.

[46] C. Adler et al. (STAR Coll.), Phys. Rev. Lett. 90 (2003) 082302.

[47] J. Adams et al. (STAR Coll.), Phys. Rev. Lett. 93 (2004) 252301. 